Research Article

\title{
Identification of Gene Mutations in Atypical Retinopathy of Prematurity Cases
}

\author{
Yian Li $\mathbb{D},{ }^{1}$ Jiakai Li, ${ }^{2}$ Xiang Zhang, ${ }^{2}$ Jie Peng $\mathbb{D},{ }^{2}$ Jing Li $\left(\mathbb{D},{ }^{2}\right.$ and Peiquan Zhao $\mathbb{D}^{2}$ \\ ${ }^{1}$ Department of Ophthalmology, Children's Hospital of Fudan University, Shanghai, China \\ ${ }^{2}$ Department of Ophthalmology, Xinhua Hospital Affiliated to Shanghai Jiao Tong University School of Medicine, \\ Shanghai, China \\ Correspondence should be addressed to Peiquan Zhao; zhaopeiquan@xinhuamed.com.cn
}

Received 27 April 2020; Accepted 7 July 2020; Published 20 August 2020

Guest Editor: Stephen T. Armenti

Copyright ( 2020 Yian Li et al. This is an open access article distributed under the Creative Commons Attribution License, which permits unrestricted use, distribution, and reproduction in any medium, provided the original work is properly cited.

\begin{abstract}
Purpose. We have observed that some preterm infants whose fundus appears very similar to eyes with familial exudative vitreoretinopathy (FEVR) present with atypical retinopathy of prematurity (ROP). To establish a definitive diagnosis and explore the possible genetic mechanism of atypical ROP, we performed gene sequencing of these cases using next-generation sequencing technology. Methods. A retrospective review of infants who presented with atypical ROP from October 2013 to February 2017 was performed. The data included gender, gestational age at birth, birth weight, family history, systemic disorders, and age-appropriate ophthalmic examinations. Fundus fluorescein angiography (FFA) of the parents was also performed. Peripheral blood was collected from the patients and their parents to sequence genes. Gene mutations were analysed. Results. Genetic testing revealed that 9 infants had FEVRrelated disease-causing gene mutations. Nine gene mutations were detected; 5 had already been reported, and the other 4 were novel. In the 18 eyes of these 9 patients, 9 eyes exhibited severe ROP. 5 cases had a positive family history. Conclusions. Gene mutations of lowdensity-lipoprotein receptor-related protein 5(LRP5), frizzled-4(FZD4), Norrie disease protein (NDP), and tetraspanin-12(TSPAN12) may play a role in the pathogenesis of ROP and cause atypical ROP or preterm FEVR. The fundus lesions of ROP patients with diseasecausing gene mutations were more serious. ROP cases should be carefully differentiated from preterm FEVR cases.
\end{abstract}

\section{Introduction}

Retinopathy of prematurity (ROP) is a complex retinal vascular disorder, and its phenotype has many similarities with familial exudative vitreoretinopathy (FEVR). Both diseases are based on abnormal development of retinal blood vessels, so there are secondary retinal complications, including retinal folds and detachment. Retinopathy in premature infants can be regarded as the arrest of normal development of retinal nerve and blood vessels, and its final pathological compensatory mechanism leads to abnormal retinal vascularisation [1]. FEVR is a disorder of retinal blood vessel development principally affecting retinal angiogenesis, leading to incomplete vascularisation of the peripheral retina and poor vascular differentiation [2]. However, emerging genetic research and fluorescein angiographic evidence suggest a margin of overlap between the two [3].
Traditionally, when clinical manifestations are difficult to diagnose, the only strategy to distinguish between diseases has been through clinical history. ROP is a disease that occurs in premature infants and generally has no genetic predisposition. However, FEVR tends to be inherited and can be observed in affected infants at full term. In clinical work, we have found a series of atypical ROP cases appearing very similar to FEVR. To confirm the diagnosis and explore the genetic mechanism of atypical ROP, we used secondgeneration sequencing technology to carry out gene detection in these cases.

\section{Materials and Methods}

This retrospective study was based on the tenets of the Declaration of Helsinki. Written informed consent was obtained from all patients or their guardians. From October 
2013 to February 2017, data were collected for infants who presented with atypical ROP at the Xinhua Hospital Affiliated to Shanghai Jiao Tong University. Next-generation sequencing technology (NGS; MyGenostics, Beijing, China) was used to perform gene sequencing in these cases. We identified a series of ROP patients $(n=9)$ with FEVR-associated mutations.

Data were collected for gender, race, gestational age (GA) at birth, birth weight (BW), dates of fundus fluorescein angiography (FFA), etc. The tests used in the study were performed before treatment.

We followed the methods of our team's FEVR-related topic [4]. All probands were native Chinese individuals. Probands and their relatives underwent comprehensive ageappropriate ophthalmic examinations including intraocular pressure (IOP) measurement, type-B ultrasound, indirect ophthalmoscopy with a 20D noncontact lens (Volk, Mentor, America), and FFA or colour fundus (RetCam 3, America), Heidelberg HRA2 (Heidelberg Company, Heidelberg, Germany), or wide-field fundus (Optomap 200Tx; Optos PLC, Dunfermline, UK) photography. The fundus findings of each proband and his or her relatives were assessed by an experienced retinal disease specialist. Any relative with ROP/FEVR-like fundus was considered to have a positive family history. Disease severity was further assessed and classified in accordance with the ROP staging system described in Hellstrom et al [1].

Peripheral blood samples were collected from all family members who signed the consent from. NGS genetic analysis was carried out on frizzled-4 (FZD4), low-density-lipoprotein receptor-related protein 5 (LRP5), Norrie disease protein $(N D P)$, tetraspanin-12 (TSPAN12), and zinc finger protein 408 (ZNF408), which are known to be related to FEVR (kinesin family member 11 (KIF11) and the $\beta$-catenin gene (CTNNB1) were not screened in this study because of their low incidence in FEVR). The variants previously reported in the Human Gene Mutation Database (HGMD; http://www. biobinternational.com/product/hgmd) were identified as pathogenic. The public databases were used to filter loci with allele frequencies (MAFs) greater than 0.05 , and the candidate pathogenic mutations were those that led to a change in the gene structure or an amino acid. The pathogenicity of candidate mutations was evaluated by computer simulation prediction. Then, the criteria of the American College of Medical Genetics and Genomics (ACMG) Standards and Guidelines [5] were used to analyse the pathogenicity. Finally, the candidate pathogenic mutations were analysed. The fundus features of ROP patients with mutations were further studied and compared with those of patients with FEVR. The characteristics of these atypical ROP cases were summarized.

\section{Results}

The demographic data of the 9 patients in this study are summarized in Table 1. Eight patients were male, and 1 was female. GA at birth ranged from 28 weeks to 36 weeks (mean: 33.78 weeks), and BW ranged from 1050 grams to 3500 grams (mean: 2324.44 grams).
TABLE 1: Baseline demographic data.

\begin{tabular}{lccc}
\hline Patient & Sex & GA at birth (wks) & BW $(\mathrm{g})$ \\
\hline 1 & M & 29 & 1050 \\
2 & M & 28 & 1270 \\
3 & M & 36 & 2950 \\
4 & M & 35 & 2500 \\
5 & M & 34 & 2250 \\
6 & M & 34 & 2200 \\
7 & F & 36 & 2500 \\
8 & M & 36 & 3500 \\
9 & M & 36 & 2700 \\
\hline
\end{tabular}

M, male; F, female; GA, gestational age; BW, birth weight.

All patients had binocular involvement. Among the 18 eyes in the 9 cases, 5 eyes (27.78\%) were stage 1, 3 (16.67\%) were stage $4 \mathrm{a}, 4(22.22 \%)$ were stage $4 \mathrm{~b}$, and $6(33.33 \%)$ were stage 5 . We defined "severe ROP" as any case at stage 3 . Severe ROP developed in 13 eyes (72.22\%) (Table 2).

Stage 1 lesions were mostly located in zone 2 without plus disease. As observed in the angiogram, irregular sprouts of vascularisation at the vascular/avascular junction, distinct pruning of vessels, and pinpoint areas of hyperfluorescence were observed (Figure 1). In addition, vascular loops with tangles beyond the edge of vascularisation were also noted in one eye (Figure 2). We did not observe typical stage 3 lesions in our cases. Stage 4a lesions were all accompanied by macular dragging (Figure 3). Stage $4 \mathrm{~b}$ lesions mostly manifested as falciform folds of the retina (Figure 4). Four stage 5 eyes had funnel-form retinal detachment associated with a flat anterior chamber (Figure 5), while the other two stage 5 eyes exhibited total retinal detachment with fibrosis and haemorrhage in front of the optic nerve (Figure 6).Nine variants were found in all the patients (Table 3). We identified 6 known mutations and 3 novel variants [6-10]. Among these, LRP5 and NDP mutations were the most common (3/9, 33.33\%), followed by mutations in FZD4 (2/9, $22.22 \%)$ and TSPAN12 (1/9, 11.11\%). All NDP mutations were hemizygous, and the other mutations were heterozygous. At least one computer simulation prediction of each novel variant was "damaging," "probably damaging," or "disease-causing," and the allelic frequency (AF) of these variants was very low $(\mathrm{N} / \mathrm{A}$ or $<0.05)$ in all databases that we accessed. Therefore, we considered these 3 novel variants (c.2431A > G and c.1434G > A in LRP5 and c.194C > T in TSPAN12) to be likely pathogenic gene mutations. However, obvious ascertainment bias for sporadic cases could not be completely excluded. Further studies to determine the function of these loci are needed to prove this theory.

Of the 9 patients, $5(55.56 \%)$ had a positive family history. NDP mutations were paternally inherited. Of the other mutations, four were maternally inherited, and two were paternally inherited.

All patients had no bone, hearing, or other anomalies except for prematurity.

\section{Discussion}

The characteristic of FEVR is failure of peripheral retinal vascularisation during ophthalmic development, which is 
TABLE 2: Clinical characteristics of 9 patients and the family members' fundus.

\begin{tabular}{lccccc}
\hline ID & Stage $(\mathrm{R} / \mathrm{L})$ & IOP $(\mathrm{mmHg} / \mathrm{L})$ & Father & \multicolumn{2}{c}{ Family history } \\
Mother & Other members \\
\hline 1 & $1 / 1$ & $12 / 10$ & - & - & - \\
2 & $4 \mathrm{a} / 4 \mathrm{a}$ & $6 / 6$ & - & - & N/A \\
3 & $4 \mathrm{a} / 1$ & $15 / 15$ & + & + & N/A \\
4 & $5 / 4 \mathrm{~b}$ & $15 / 6$ & - & + & N/A \\
5 & $5 / 5$ & $8 / 8$ & - & - & N/A \\
6 & $5 / 5$ & $23 / 11$ & - & N/A \\
7 & $1 / 5$ & $18 / 10$ & - & - & N/A \\
8 & $4 \mathrm{~b} / 1$ & $9 / 8$ & - & + & Brother, - \\
9 & $4 \mathrm{~b} / 4 \mathrm{~b}$ & $20 / 13$ & N/A \\
\hline
\end{tabular}

IOP, intraocular pressure; N/A, not applicable; R, right eye; L, left eye; -, normal; +, abnormal fundus.

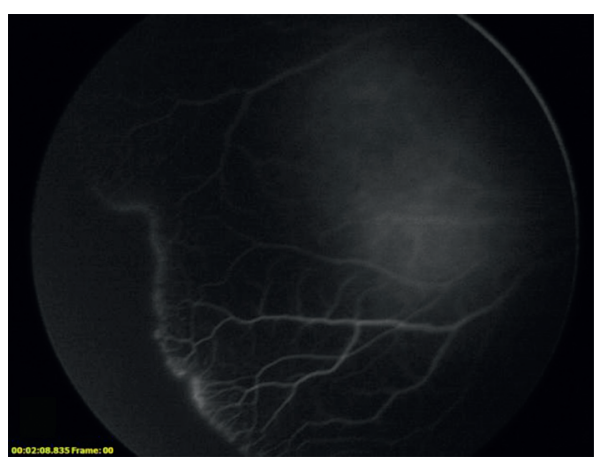

(a)

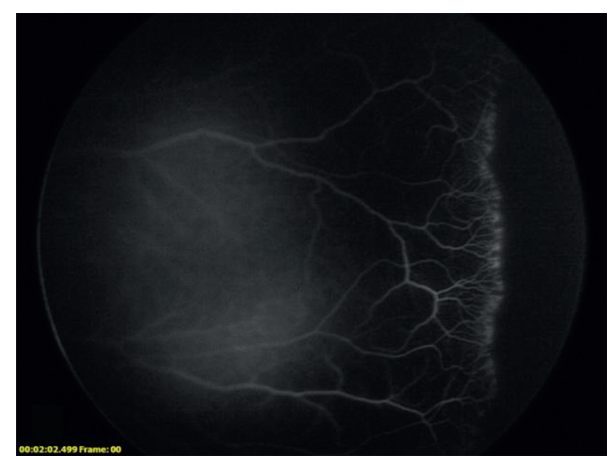

(b)

Figure 1: Fluorescein angiography (FA) of case 1. (a) Right eye. (b) Left eye. Both eyes were zone II stage 1 without plus disease. Irregular sprouts of vascularisation at the vascular/avascular junction, distinct pruning of vessels, and pinpoint areas of hyperfluorescence were observed.

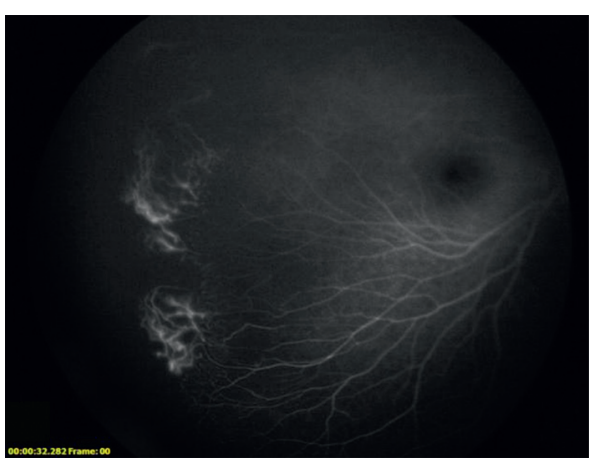

(a)

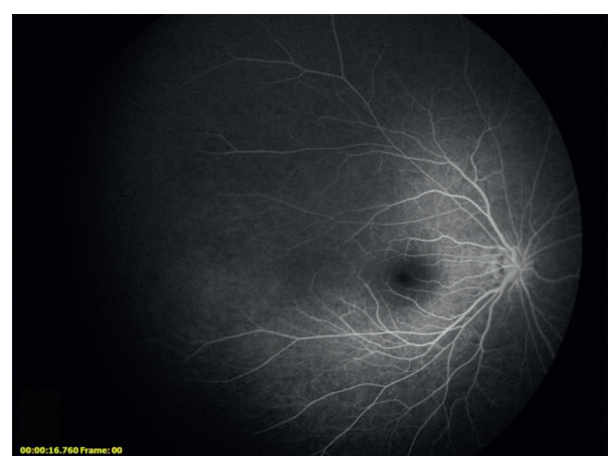

(b)

Figure 2: Left eye of case 7. Vascular loops with tangles beyond the edge of vascularisation were observed.

associated with a spectrum of clinical manifestations. FEVR can be confirmed by genetic testing [11]. To date, five causative genes have been discovered to be related to FEVR, including LRP5, FZD4, TSPAN12, NDP, and ZNF408. FEVR has Mendelian inheritance [2, 11-14]. ROP is a multifactorial disease that is genetically heterogeneous with multiple alleles with varying magnitudes of effect. The similarity of clinical manifestations between ROP and FEVR further reflects the possibility of the involvement of these genes in ROP pathogenesis.

Recently, several genetic analyses have shown that FEVRrelated mutations exist in children with advanced ROP [15-21]. The clinical manifestations of these two diseases are very similar. Both diseases can present with peripheral avascularity, neovascularisation, vitreous haemorrhage, subretinal exudation, vascular dragging, radial retinal folds, and tractional 


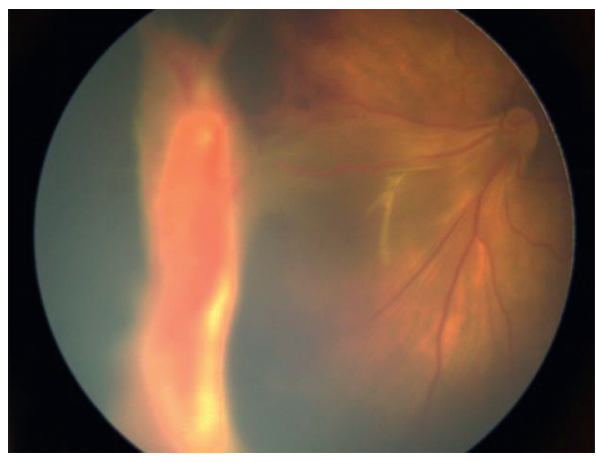

(a)

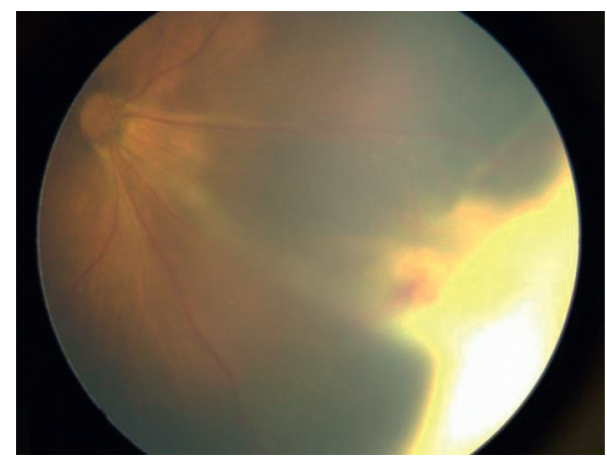

(b)

FIgURE 3: Colour fundus of case 2. (a) Right eye. (b) Left eye. Both eyes were stage 4a accompanied by macular dragging.

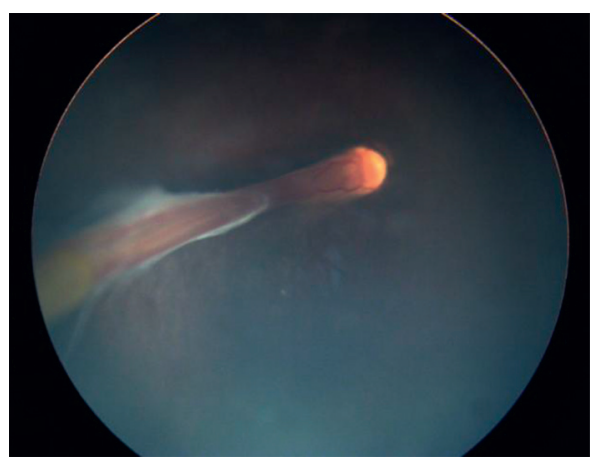

(a)

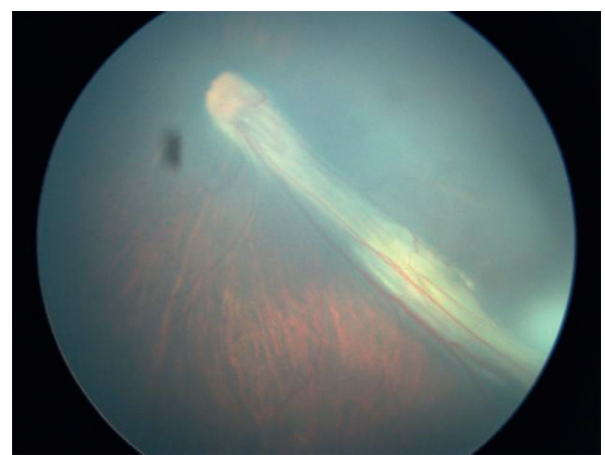

(b)

Figure 4: (a) Right eye fundus of case 8. (b) Left eye fundus of case 9. Stage 4 b lesions were mostly manifested as falciform fold of the retina.

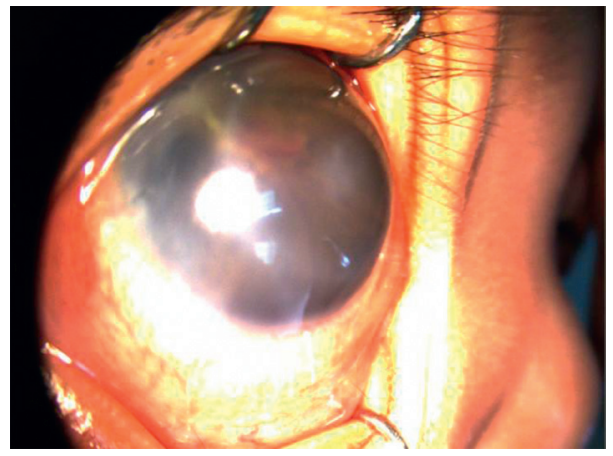

(a)

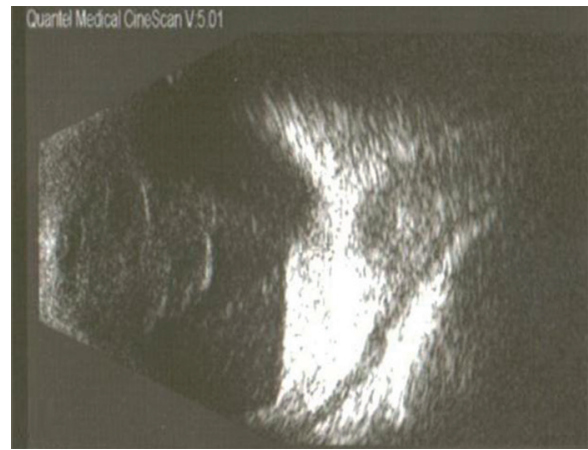

(b)

Figure 5: Right eye of case 4. (a) Flat anterior chamber. (b) B-scan of the right eye showed funnel-form retinal detachment.

retinal detachments [22-25]. Furthermore, in fundus FA, both FEVR and ROP were thought to appear as abrupt cessation of the capillary network with the formation of scalloped borders and leakage of fluorescein dye from this border [3].

Some scholars believe that most ROP patients display high-fluorescence lesions similar to "popcorn," abnormal branches (such as circumferential or tangled vessels), local telangiectasis, neovascularisation, and leakage [3]. However, the characteristics of the vascular/avascular junction in
FEVR patients include a sudden cessation of capillaries, bulbous vascular endings, venous/venous shunting (rather than arterio/venous), and abnormal increased branches of vessels [26]. In addition, irregular sprouts of vascularisation were observed at the vascular/avascular junction in patients with FEVR in contrast to the vessel profile of classical ROP patients, which was more uniform [3]. Angiography of stage 1 patients in our series showed that they were more likely to have FEVR rather than typical ROP. Only one case (Case 7) 


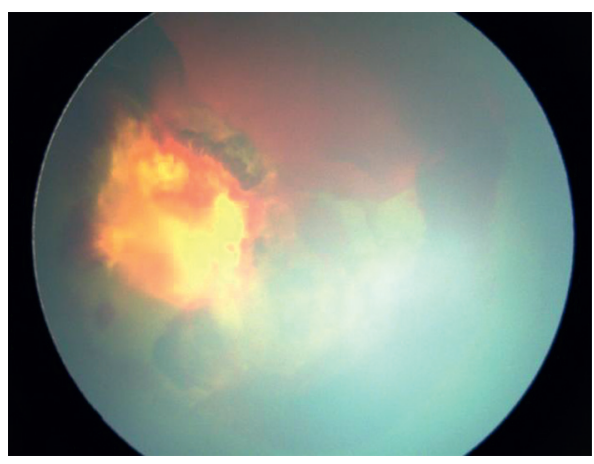

(a)

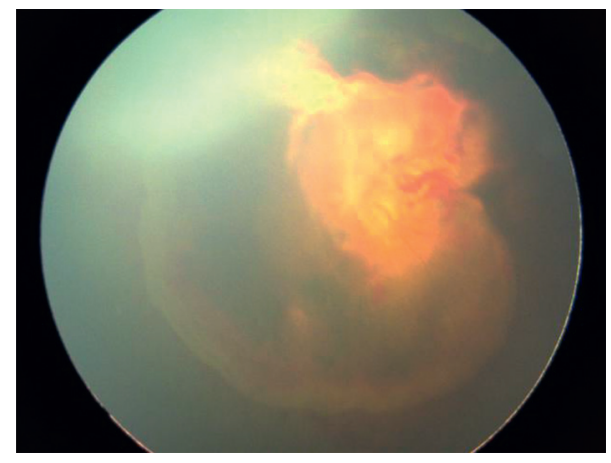

(b)

FIgURE 6: Colour fundus of case 5. (a) Right eye. (b) Left eye. Both eyes showed total retinal detachment with fibrosis and haemorrhage in front of the optic nerve.

showed vascular loops with tangles, which are more inclined to ROP. Nevertheless, ROP is difficult to distinguish from FEVR simply by clinical manifestations.

All nine cases except for two (cases 1 and 2) had a GA of 34 weeks or more and a minimum BW of 2200 grams. China's ROP screening standards are a BW $<2000$ grams and a GA $<32$ weeks. The GAs and BWs of these seven infants were significantly greater than those of the infants with typical ROP. Furthermore, in four cases (cases 3, 7, 8, and 9), the GAs were 36 weeks, which is close to full term. Vascularisation of the retina begins at approximately 18 weeks of gestation and is complete by $38-40$ weeks of gestation [27]. ROP represents the pathological compensatory mechanism of retinal abnormal vascularisation in premature infants due to the arrest of normal development of retinal nerves and vessels. Greater immaturity at birth corresponds to a more aggressive pathological response later. Both a young GA and a low BW are associated with increased severity of ROP [28]. In both animal and human studies, hyperoxia is an important factor that inhibits ROP angiogenesis [29-31]. Studies have shown that among infants with a GA of less than 27 to 29 weeks, ROP was reported in $33 \%$ to $73 \%$ of infants and severe ROP was reported in $10 \%$ to $35 \%$ of infants [32-37]. Typical ROP occurs in young-GA and low-BW infants. Of the 14 eyes in the 7 cases, 11 eyes (78.57\%) had severe lesions, suggesting that the severity of the lesions may be affected not only by the environment, GA, and BW but also by gene mutations.

Case 1 was born at a GA of 29 weeks with a BW of $1050 \mathrm{~g}$, and both eyes were zone II stage 1 without plus disease. The case was not serious, and according to the characteristics of FFA, we considered this case to be similar to FEVR. Case 2 was born at a GA of 28 weeks with a BW of $1270 \mathrm{~g}$. Both eyes were stage $4 \mathrm{a}$ with temporal tractional retinal detachment, proliferation, exudation, and macular dragging. This case is more similar to a typical ROP.

John [3] reported nine cases of premature delivery with features resembling FEVR and introduced a new classification: ROPER (ROP vs. FEVR). However, we believe that these cases cannot be simply classified as one disease. Some cases may be premature FEVR, while others may be ROP combined with FEVR, especially those with more severe disease, which we may call "ROPER." Family history and genetic testing also support the diagnosis of FEVR. Mutations of FEVR-related pathogenic genes have been investigated in ROP. In one study, mutations in the FZD4 gene were found in up to $7.5 \%$ of patients with severe ROP [38-41]. A study of 421 patients with various vitreoretinopathies found significant correlations between the FZD4 double missense mutation and both ROP and FEVR [41]. In a study of 53 Japanese ROP patients, $13 \%$ of advanced ROP (stage 4 or 5 ) patients were found to carry mutations in FZD4 or LRP5 [40]. According to an American study, $11 \%$ of infants with severe ROP had polymorphisms in the NDP gene [20]. These studies indicate that the presence of FEVR mutations in ROP may contribute to more advanced retinopathy, which supports our point that ROP with FEVR mutation is more severe, possibly because of ROP combined with FEVR. One hypothesis is that advanced ROP is caused by mutations of genes with less severe functional impact, while FEVR is caused by mutations with greater phenotypic consequences [40]. Recently, an Indian study found a heterozygous variant in the TSPAN12 gene in a patient with threshold ROP [42]. However, a limitation of these reports (including this study) is that they may have excessively relied on computer simulations to predict the pathogenicity of alleles. Functional analysis is required to determine causation more clearly. In addition, most genetic studies in ROP have been performed on candidate genes. The advantage of candidate gene studies is that in association with a disease, biologic plausibility often exists when selecting certain pathways. The limitation, however, is that only those genes that are believed to be involved are analysed. A genome-wide association study on a larger number of ROP infants is needed.

ROP cases should be carefully differentiated from preterm FEVR cases, especially ROPER and preterm FEVR. However, distinguishing the two diseases only by GA is unreasonable. ROPER presents with indeterminate activation episodes, and FEVR is relatively stable; accurate diagnosis provides guiding significance for treatment. ROPER requires close monitoring for disease progression early and often throughout life, including serial FA and treatment with cryotherapy, laser, and anti-vascular endothelial growth 


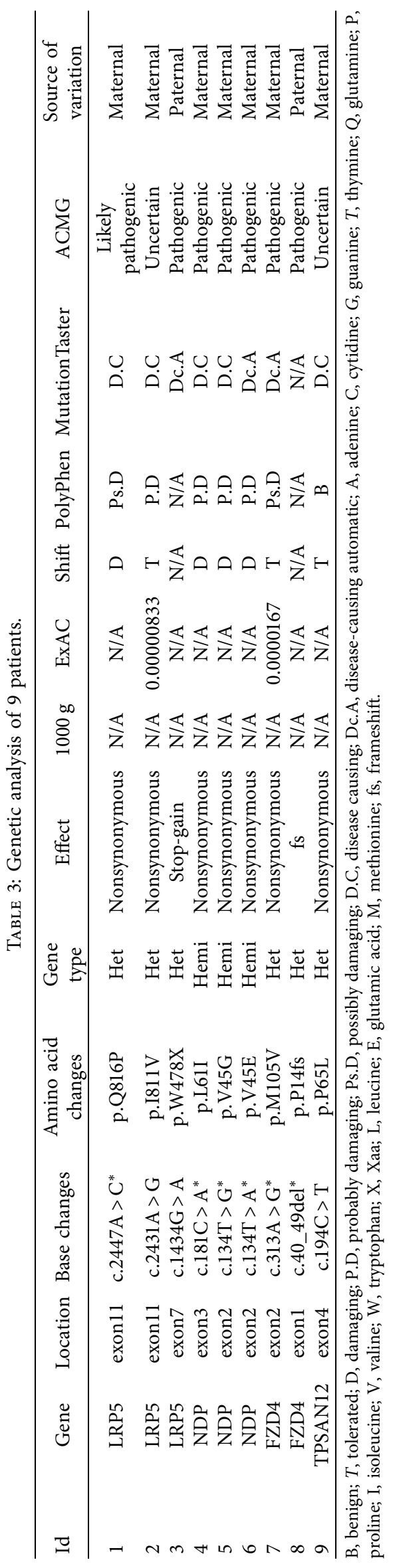


factor (anti-VEGF) injections [3]. However, only patients with FEVR with significant signs of progression or a high risk of progression should accept treatment [43].

\section{Conclusion}

ROP is a complex disease in that evidence suggests that it is influenced by both genetic and environmental factors. Our findings showed that gene mutations of LRP5, FZD4, NDP, and TSPAN12 may play a role in the pathogenesis of ROP and cause atypical ROP or preterm FEVR. The fundus lesions of ROP patients with disease-causing gene mutations are more serious and may be FEVR combined with ROP, and such diseases may be called ROPER. ROP cases should be carefully differentiated from preterm FEVR cases.

\section{Data Availability}

The data used to support the findings of this study have not been made available because of the ethical concerns and patient privacy.

\section{Disclosure}

This work has been presented as an oral report in the $23 \mathrm{rd}$ National Congress of Ophthalmology of the Chinese Medical Association.

\section{Conflicts of Interest}

The authors declare that they have no conflicts of interest.

\section{Acknowledgments}

The authors thank all participants in this study for their collaborations. The authors are grateful to the technical staff at Children's Hospital of Fudan University and Xinhua Hospital Affiliated to Shanghai Jiao Tong University School of Medicine for their assistance and to MyGenostics, Beijing, for technical support. This work was supported by the National Natural Science Foundation Project of China (Grant No. 81470642).

\section{References}

[1] A. Hellström, L. E. Smith, and O. Dammann, "Retinopathy of prematurity," The Lancet, vol. 382, no. 9902, pp. 1445-1457, 2013.

[2] D. F. Gilmour, "Familial exudative vitreoretinopathy and related retinopathies," Eye, vol. 29, no. 1, pp. 1-14, 2015.

[3] V. J. John, J. I. McClintic, D. J. Hess, and A. M. Berrocal, "Retinopathy of prematurity versus familial exudative vitreoretinopathy: report on clinical and angiographic findings," Ophthalmic Surgery, Lasers and Imaging Retina, vol. 47, no. 1, pp. 14-19, 2016.

[4] Y. Li, J. Peng, J. Li et al., "The characteristics of digenic familial exudative vitreoretinopathy," Graefe's Archive for Clinical and Experimental Ophthalmology, vol. 256, no. 11, pp. 2149-2156, 2018.

[5] S. Richards, N. Aziz, N. Aziz et al., "Standards and guidelines for the interpretation of sequence variants: a joint consensus recommendation of the American College of medical genetics and Genomics and the association for molecular pathology," Genetics in Medicine, vol. 17, no. 5, pp. 405-423, 2015.

[6] W.-C. Wu, K. Drenser, M. Trese, A. Capone Jr., and W. Dailey, "Retinal phenotype-genotype correlation of pediatric patients expressing mutations in the Norrie disease gene," Archives of Ophthalmology, vol. 125, no. 2, pp. 225-230, 2007.

[7] H. Kondo, H. Hayashi, K. Oshima, T. Tahira, and K. Hayashi, "Frizzled 4 gene (FZD4) mutations in patients with familial exudative vitreoretinopathy with variable expressivity," British Journal of Ophthalmology, vol. 87, no. 10, pp. 12911295, 2003.

[8] P. Fei, Q. Zhang, L. Huang et al., "Identification of two novel LRP5 mutations in families with familial exudative vitreoretinopathy," Molecular Vision, vol. 20, pp. 395-409, 2014.

[9] L.-Y. Jia, X. X. Li, W. Z. Yu, W. T. Zeng, and C. Liang, "Novel frizzled-4 gene mutations in Chinese patients with familial exudative vitreoretinopathy," Archives of Ophthalmology, vol. 128, no. 10, pp. 1341-1349, 2010.

[10] D. Lev, Y. Weigl, M. Hasan et al., "A novel missense mutation in theNDP gene in a child with Norrie disease and severe neurological involvement including infantile spasms," American Journal of Medical Genetics Part A, vol. 143A, no. 9, pp. 921-924, 2007.

[11] C. Toomes, H. M. Bottomley, S. Scott et al., "Spectrum and frequency ofFZD4Mutations in familial exudative vitreoretinopathy," Investigative Opthalmology \& Visual Science, vol. 45, no. 7, pp. 2083-2090, 2004.

[12] J. Robitaille, M. L. E. MacDonald, A. Kaykas et al., "Mutant frizzled-4 disrupts retinal angiogenesis in familial exudative vitreoretinopathy," Nature Genetics, vol. 32, no. 2, pp. 326330,2002

[13] K. Nikopoulos, C. Gilissen, A. Hoischen et al., "Next-generation sequencing of a $40 \mathrm{Mb}$ linkage interval reveals TSPAN12 mutations in patients with familial exudative vitreoretinopathy," The American Journal of Human Genetics, vol. 86, no. 2, pp. 240-247, 2010.

[14] R. W. J. Collin, K. Nikopoulos, M. Dona et al., "ZNF408 is mutated in familial exudative vitreoretinopathy and is crucial for the development of zebrafish retinal vasculature," Proceedings of the National Academy of Sciences, vol. 110, no. 24, pp. 9856-9861, 2013.

[15] B. S. Shastry, "Genetic susceptibility to advanced retinopathy of prematurity (ROP)," Journal of Biomedical Science, vol. 17, no. 1, p. 69, 2010.

[16] B. S. Shastry, S. D. Pendergast, M. K. Hartzer, X. Liu, and M. T. Trese, "Identification of missense mutations in the Norrie disease gene associated with advanced retinopathy of prematurity," Archives of Ophthalmology, vol. 115, no. 5, pp. 651-655, 1997.

[17] M. Hiraoka, D. M. Berinstein, M. T. Trese, and B. S. Shastry, "Insertion and deletion mutations in the dinucleotide repeat region of the Norrie disease gene in patients with advanced retinopathy of prematurity," Journal of Human Genetics, vol. 46, no. 4, pp. 178-181, 2001.

[18] S. J. Talks, N. Ebenezer, P. Hykin et al., "De novo mutations in the 5 ' regulatory region of the Norrie disease gene in retinopathy of prematurity," Journal of Medical Genetics, vol. 38, no. 12, p. E46, 2001.

[19] M. Z. Haider, L. V. Devarajan, M. Al-Essa, and H. Kumar, “A C597\&rarr;A polymorphism in the Norrie disease gene is associated with advanced retinopathy of prematurity in premature Kuwaiti infants," Journal of Biomedical Science, vol. 9, no. 4, pp. 365-370, 2002. 
[20] K. A. Hutcheson, P. C. Paluru, S. L. Bernstein et al., "Norrie disease gene sequence variants in an ethnically diverse population with retinopathy of prematurity," Molecular Vision, vol. 11, pp. 501-508, 2005.

[21] M. Hiraoka, H. Takahashi, H. Orimo, M. Hiraoka, T. Ogata, and N. Azuma, "Genetic screening of Wnt signaling factors in advanced retinopathy of prematurity," Molecular Vision, vol. 16, pp. 2572-2577, 2010.

[22] An International Classification of Retinopathy of Prematurity, "The committee for the classification of retinopathy of prematurity," Archives of Ophthalmology, vol. 102, no. 8, pp. 1130-1134, 1984.

[23] V. G. Criswick and C. L. Schepens, "Familial exudative vitreoretinopathy," American Journal of Ophthalmology, vol. 68, no. 4, pp. 578-594, 1969.

[24] H. Miyakubo, K. Hashimoto, and S. Miyakubo, "Retinal vascular pattern in familial exudative vitreoretinopathy," Ophthalmology, vol. 91, no. 12, pp. 1524-1530, 1984.

[25] W. E. Benson, "Familial exudative vitreoretinopathy," Transactions of the American Ophthalmological Society, vol. 93, pp. 473-521, 1995.

[26] A. H. Kashani, K. T. Brown, E. Chang, K. A. Drenser, A. Capone, and M. T. Trese, "Diversity of retinal vascular anomalies in patients with familial exudative vitreoretinopathy," Ophthalmology, vol. 121, no. 11, pp. 2220-2227, 2014.

[27] S. Hughes, H. Yang, and T. Chan-Ling, "Vascularization of the human fetal retina: roles of vasculogenesis and angiogenesis," Investigative Ophthalmology \& Visual Science, vol. 41 , no. 5, pp. 1217-1228, 2000.

[28] W. V. Good, R. J. Hardy, V. Dobson et al., "The incidence and course of retinopathy of prematurity: findings from the early treatment for retinopathy of prematurity study," Pediatrics, vol. 116, no. 1, pp. 15-23, 2005.

[29] M. E. Hartnett and J. S. Penn, "Mechanisms and management of retinopathy of prematurity," New England Journal of Medicine, vol. 367, no. 26, pp. 2515-2526, 2012.

[30] L. E. Smith, E. Wesolowski, A. McLellan et al., "Oxygeninduced retinopathy in the mouse," Investigative Ophthalmology \& Visual Science, vol. 35, no. 1, pp. 101-111, 1994.

[31] K. M. Connor, N. M. Krah, R. J. Dennison et al., "Quantification of oxygen-induced retinopathy in the mouse: a model of vessel loss, vessel regrowth and pathological angiogenesis," Nature Protocols, vol. 4, no. 11, pp. 1565-1573, 2009.

[32] D. Austeng, K. B. Kallen, U. W. Ewald, P. G. Jakobsson, and G. E. Holmstrom, "Incidence of retinopathy of prematurity in infants born before 27 weeks' gestation in Sweden," Archives of Ophthalmology, vol. 127, no. 10, pp. 1315-1319, 2009.

[33] T. Markestad, P. I. Kaaresen, A. Ronnestad et al., "Early death, morbidity, and need of treatment among extremely premature infants," Pediatrics, vol. 115, no. 5, pp. 1289-1298, 2005.

[34] K. Allegaert, K. de Coen, H. Devlieger, and G. EpiBel Study, "Threshold retinopathy at threshold of viability: the EpiBel study," British Journal of Ophthalmology, vol. 88, no. 2, pp. 239-242, 2004.

[35] B. A. Darlow, J. L. Hutchinson, D. J. Henderson-Smart et al., "Prenatal risk factors for severe retinopathy of prematurity among very preterm infants of the Australian and New Zealand Neonatal Network," Pediatrics, vol. 115, no. 4, pp. 990-996, 2005.

[36] C. Weber, M. Weninger, K. Klebermass et al., "Mortality and morbidity in extremely preterm infants (22 to 26 weeks of gestation): Austria 1999-2001," Wiener Klinische Wochenschrift, vol. 117, no. 21-22, pp. 740-746, 2005.
[37] V. Tommiska, K. Heinonen, L. Lehtonen et al., "No improvement in outcome of nationwide extremely low birth weight infant populations between 1996-1997 and 19992000," Pediatrics, vol. 119, no. 1, pp. 29-36, 2007.

[38] M. MacDonald, Y. Goldberg, J. Macfarlane, M. Samuels, M. Trese, and B. Shastry, "Genetic variants of frizzled-4 gene in familial exudative vitreoretinopathy and advanced retinopathy of prematurity," Clinical Genetics, vol. 67, no. 4, pp. 363-366, 2005.

[39] A. Ells, D. L. Guernsey, K. Wallace et al., "Severe retinopathy of prematurity associated withFZD4mutations," Ophthalmic Genetics, vol. 31, no. 1, pp. 37-43, 2010.

[40] H. Kondo, S. Kusaka, A. Yoshinaga, E. Uchio, A. Tawara, and T. Tahira, "Genetic variants of FZD4 and LRP5 genes in patients with advanced retinopathy of prematurity," Molecular Vision, vol. 19, pp. 476-485, 2013.

[41] W. A. Dailey, W. Gryc, P. G. Garg, and K. A. Drenser, "Frizzled-4 variations associated with retinopathy and intrauterine growth retardation," Ophthalmology, vol. 122, no. 9, pp. 1917-1923, 2015.

[42] S. Rathi, S. Jalali, G. R. Musada et al., "Mutation spectrum of NDP, FZD4 and TSPAN12 genes in Indian patients with retinopathy of prematurity," British Journal of Ophthalmology, vol. 102, no. 2, pp. 276-281, 2018.

[43] D. Shukla, J. Singh, G. Sudheer et al., "Familial exudative vitreoretinopathy (FEVR). Clinical profile and management," Indian Journal of Ophthalmology, vol. 51, no. 4, pp. 323-328, 2003. 